\title{
Direct Digital Manufacturing of Architectural Models using Binder Jetting and Polyjet Modeling
}

\author{
Stefan Junk ${ }^{1}$, Michelle Niederhüfner ${ }^{2}$, Nina Borkowska ${ }^{3}$, \\ Steffen Schrock ${ }^{4}$ \\ ${ }^{1,2,4}$ University of Applied Sciences Offenburg, Department of Business and Engi- \\ neering, Campus Gengenbach, Germany ${ }^{3}$ IFP 2 Hangs GmbH, Engineering office \\ for planning and project management, Lahr, Germany \\ 1,4\{stefan.junk|steffen.schrock\}@hs-offenburg.de²mniederh@stud.hs-offenburg. \\ de n.borkowska@ifp2.de
}

\begin{abstract}
Today, architectural models are an important tool for illustrating drawn-on plans or computer-generated virtual models and making them understandable. In addition to the conventional methods for the manufacturing of physical models, a wide range of processes for Direct Digital Manufacturing (DDM) has spread rapidly in recent years. In order to facilitate the application of these new methods for architects, this contribution examines which technical and economic results are possible using $3 D$ printed architectural models. Within a case study, it will be shown on the basis of a multi-storey detached house, which kind of data preparation is necessary. The DDM of architectural models will be demonstrated using two widespread techniques and the resulting costs will be compared.
\end{abstract}

Keywords: Architeetual model, CAAD, Direct Digital Manufacturing, Binder Jetting, Polyjet Modelling

\section{INTRODUCTION}

Architectural models have been an important architect's work and design tool for centuries to illustrate two-dimensional designs as 3D sculptural objects. Using design models, architects and builders can quickly understand and evaluate the cubature of a design as well as the spatial relationships. Especially for nonprofessionals, who are inexperienced in the reading of architectural drawings, a model is often more vivid than two-dimensional representations as sketches. In contrast to architectural representations as virtual reality, e.g. with the aid of largescale screens (i. e. powerwall) or virtual glasses, the architectural model provides direct and haptic access to the design. It is advantageous that for viewing no time-consuming data preparation by rendering and no cost-intensive hardware (i. e. projector, VR glasses) is needed.

Architectural models can be manufactured using a number of conventional manufacturing techniques. Thus, various model parts are made of plastic metal, wood, glass, paper or even plaster. The manufacturing takes place indirectly with elaborate milling or cutting machines that are equipped with different tools and also need to be programmed. Subsequently, complex assembly work is usually necessary 
to connect the different model parts and materials . For some years, therefore, the methods of Additive Manufacturing (AM) or Direct Digital Manufacturing (DDM, shortly: 3D printing) have been used extensively in the production of architectural models. In addition to the direct manufacturing, these methods also offer the advantage that complex and curved freeform geometries can be implemented.

The basis for AM is always a $3 \mathrm{D}$ virtual model created using CAAD software. This model is then checked during data preparation for printability and redesigned considering particular design guidelines and restrictions. Subsequently, the 3D printing can be done. A major obstacle to the further spread of additive manufacturing is that many architects do not have the necessary knowledge for data processing. In addition, especially in small architect's offices still mainly 2D CAAD software is used, so that a $3 D$ model for additive manufacturing is not available. As a result, the $3 \mathrm{D}$ models have to be extensively redesigned from the 2D designs (such as floor plans and façade plans). In this article it should therefore be analyzed how a 3D model can be transferred directly from the CAAD data to additive manufacturing.

Also for the additive manufacturing of the architectural models, a large number of methods are available today, e.g. models are made of plaster, plastic, metal or paper. In this contribution, two of the most important methods for the production of architectural models, namely Binder Jetting (BJ: printing material: gypsum-plastic powder with liquid adhesive) and the Polyjet Modeling (PJM: printing material: UVlight-curing photopolymer) are considered in more detail. This clarifies the technical and economic differences arising from the use of different 3D printing methods and materials.

\section{LITERATURE REVIEW}

The development of additive processes began more than 30 years ago with a patent for stereolithography. Since then, many different processes have been developed and put on the market. All methods have in common that the model are built layer by layer. Like- wise, in all processes, the layers are built directly from the CAAD data without additional aids such as tools or clamping devices. The market research by Wohlers et al. (2017) show high growth rates for years both in the sale of 3D printers and in the number of additively produced models. For two decades, additive manufacturing has also been used to produce architectural models. Thus, the studies of Ryder et al. (2002) showed that the additive methods could be usefully used despite the high costs involved in the implementation of architectural models. However, according to Gibson et al. (2002) the 3D-printed model were still only limited usable due to the small available size and the defective appearance.

Since then, the technology has evolved significantly. As Wong and Hernadez (2012) show, today printers with a much larger space are available. In addition, today there are systems on the market that can produce both monochrome and polychrome models. According to Stavric et al. (2013) can architectural models thus be created by additive methods as well as by reverse engineering. Even partially transparent models can be created today. In order to find a suitable additive manufacturing process for architectural models, Mancanares et al. (2014) developed a catalog with important criteria. The benefits of digital manufacturing become particularly apparent when complex shapes (e.g. filigree structures or organic shapes) are to be modeled. Zerdad and Paulino (2014) impressively demonstrate the application of digital manufacturing for the production of models with organic shapes in the topology optimization of bridges.

As Hull and Willet (2017) show, the 3D-printed models can be used meaningfully for different functions in all areas of the development process, from the first draft via concept studies to the presentation model. By physically mapping of large quantities of virtual CAAD data, the big data can be better represented and made more comprehensible. Implementing models at different scales using parameters as well as mapping different textures and materials using Digital Materials is demonstrated by Junk and 
Figure 1

Design of the family house designed using CAAD-system Archicad
Gawron (2018). However, as Meijs (2014) explains, the reality is different. Many architects are still hesitant to make the leap to $3 \mathrm{D}$ printing. In the daily practice in architecture offices, cost and complexity are usually the biggest hurdles.

\section{METHODOLOGY}

There are still some challenges that prevent the introduction of Direct Digital Manufacturing in the production of architectural models, especially in small and medium-sized architectural firms. One of the technical challenges is to find a suitable method for architectural models out of the large number of available AM methods. In addition, requirements for data preparation for 3D printing are unknown. However, there are also economic obstacles. This means that in many cases the time required for data preparation can only be estimated poorly. Furthermore, the cost of a model are unknown. Similarly, the cost differences for different AM methods are difficult to assess.

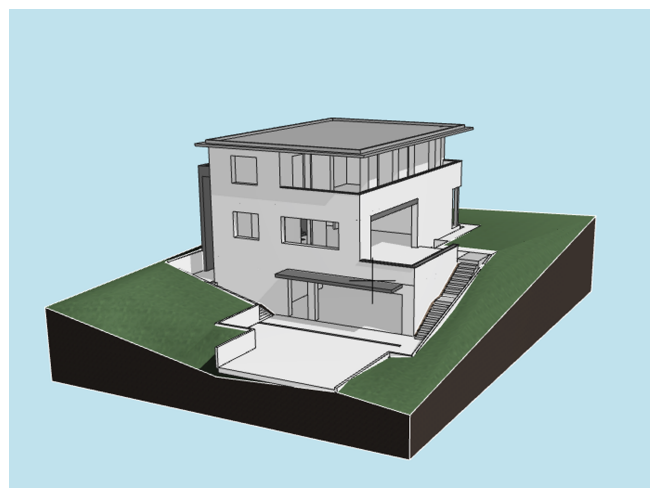

Therefore, this contribution demonstrate how to prepare the data from 3D-CAAD for 3D printing. In a case study, a one-family detached house as a typical project for an architecture firm of small to medium size is chosen. In order to investigate the additive methods with regard to their potential for the production of three-dimensional architectural models, a project from the architects office $\mathrm{IFP}^{2}$ will be made available and then $3 \mathrm{D}$ printed in a print-ready manner. The 3D CAAD dataset is then produced using two different $3 D$ printing technologies to compare the procedures. The CAAD modell represnts detached house with the dimensons of $22.27 \times 15.31 \times 9.98$ meters, which consists of four floors. The house is divided into a basement, ground floor, upper floor and attic. The CAAD model also contains a 3D terrain model, which measures $28.99 \times 19.94 \times 6.92$ meters. The widely used ArchiCAD software is used for design and data preparation.

Whitin the case study is analyzed which elements from CAAD are transferred to $3 D$ printing and which elements can not be displayed due to particular restrictions (e.g. insufficient wall thickness). Subsequently, the data processing takes place in preprocessing of the printer software. To do this, the easily accessible software, e.g. or Mircosoft "3D Builder" or Autodesk "Netfabb", is used. Here, the feasibility check and the setting of specific 3D printing parameters are carried out. This model, as shown in Figure 1 , is the result of a design in the CAAD program Archicad and is printed at a scale of 1:100 in the experimental procedure. In consultation with the company IFP' ${ }^{2}$, the model is manufactured at the University of Applied Sciences Offenburg using the methods Binder Jetting and PolyJet Modeling.

\section{DATA PREPARATION IN PRE-PROCESSING}

The first step of the data preparation carried out using a CAAD program. Once the formal requirements have been met, the 3D model must be transferred to a repair software in order to carry out further necessary reprocessing. Finally, the simplified and also checked model is transferred for Direct Digital Manufacturing in the 3D printer software. This process chainis illustrated in Figure 2.

In order to be able to produce the 3D data of the architectural models by means of 3D printing, it is important to first display only the relevant components and to hide furniture, plants and other facilities. In addition, it is also of great importance to determine the allowable size of the resulting 3D physical model. If the model should then be too large, 
Virtual Model

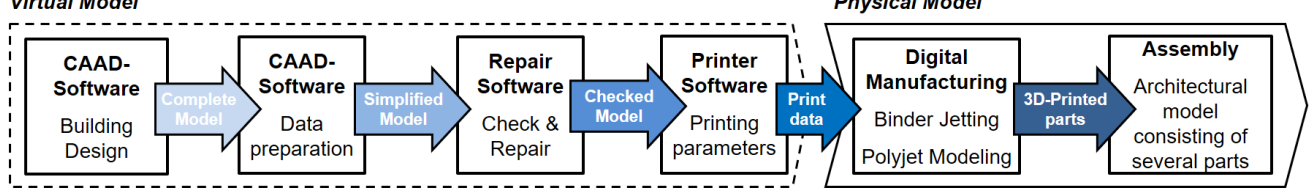

one can separate it using one or more sections. In addition, requirements for the wall thicknesses and a "solid model" are important features that must be considered in the preparation in general.

Since downsizing from an original model tends to make the walls, columns, railings, windows, and doors too thin, an appropriate adjustment must be made to the model file. It is therefore important to provide the model with sufficient wall thickness or to thicken certain walls so that the minimum requirements of the respective printer can be met. Finally, to use a 3D Archicad model for 3D printing, the model must consist of one component or "solid model". As long as the individual components are only linked with each other, they also remain different components.

\section{DIGITAL MANUCATURING}

The digital manufacturing of the models uses two different, easily accessible 3D printing methods. On the one hand, Binder Jetting (BJ) is used, which works with a simple mineral building material and offers a high 3D printing speed. The 3D printer used in this

\section{Virtual Model}

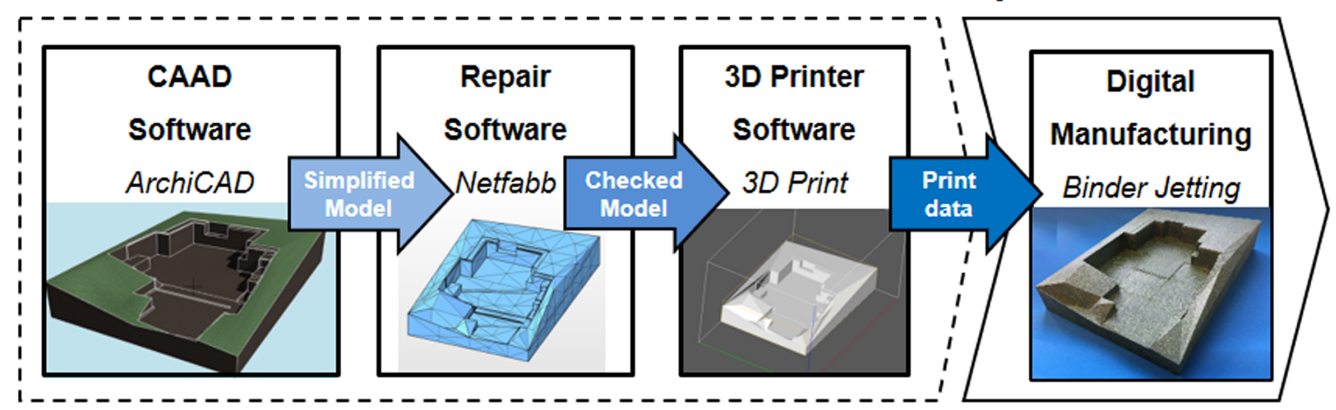
the Polyjet Modeling (PJM) process, which is char-
Figure 2

Process for Direct Digital Manufacturing of Architectural Models form CAAD to 3D model

contribution (Projet660 Pro by 3D-Systems) is a fullcolor printer with a build size of $254 \times 381 \times 203 \mathrm{~mm}$. First, a powder bed is made of a polymer gypsum powder, to which then a binder and, if necessary, also colour is sprayed on with the help of nozzles. The 3D printer advertises with its high resolution of $600 \times 540 \mathrm{dpi}$, uses environmentally friendly materials and is according to the manufacturer for architects the ideal choice. This makes it possible to produce photorealistic models through precise and consistent color gradations. It offers a fast print speed, which can be accelerated up to $28 \mathrm{~mm} / \mathrm{h}$ by the use of "stacking functions" and choosing the printing mode "monochrome".

On the other hand, this case study also uses acterized by high $3 \mathrm{D}$ printing quality as well as long 3D printing times. The 3D color printer (J750

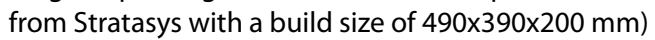
can create over 500,000 colors with a resolutiion of $600 \times 600 \times 1800 \mathrm{dpi}$. The structure is achieved by the direct jetting of a photopolymer, which is cured with UV light. A special feature here is that it can be
Figure 3

Application of the process chain for the Direct Digtial Manufacturing of a terrain modell 
printed transparently. The 3D printer can process up to six materials at the same time and guarantee a high level of detail with layer thicknesses of only 14 microns as well as a smooth surface. The the small layer thickness results in relatively slow speed of 80 grams per hour.

This process chain is explained using the example of the terrain model representing the construction site (see Fig. 3). In this example, the 3D terrain model is first exported in STL format at a 1:100 scale from Archicad, that represents the target state. Subsequently, this file is opened in the two differnt repair software programs "3D Builder" and "Netfabb" and then described by screenshots. By subsequent transfer in the printer software programs "3D Print" (BJ) and "GrabCAD" (PJM), the model shows some more edges in comparison to the target state. However, there is no serious difference between the transferred files and the target state.

Figure 4

Comparsion of technincal results form Binder Jetting and Polyjet Modeling

\section{TECHNICAL RESULTS}

With regard to the technical results, the building was printed once each in color and in monochrome using BJ and once in monochrome using the PJM process. In addition, the colored building is imported for testing purposes in the printing software "GrabCAD". To do this, the model is first exported as STL (geometry only), as well as VRML file (geometry and texture) and then edited or repaired in Netfabb. In all cases, good $3 \mathrm{D}$ printing results and a high qualitiy of appearence were achieved. Also, the terrain model is then printed once in monochrome and once in color mode. Before, the colored terrain was colored and textured via Netfabb.

\section{ECONOMIC CONSIDERATIONS}

A comparison of the methods reveals the significant economic differences of the two AM methods for use in the production of architectural models. The costs of printing, materials and personnel are considered

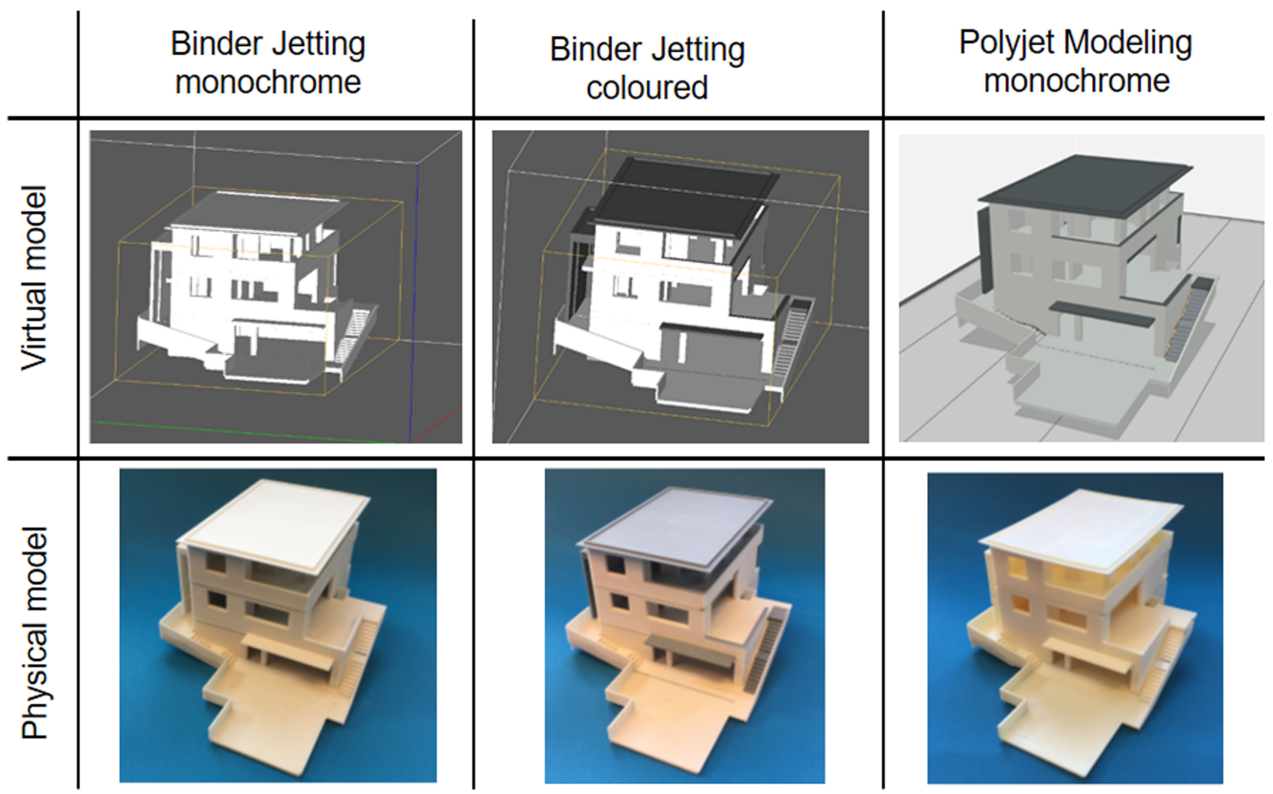




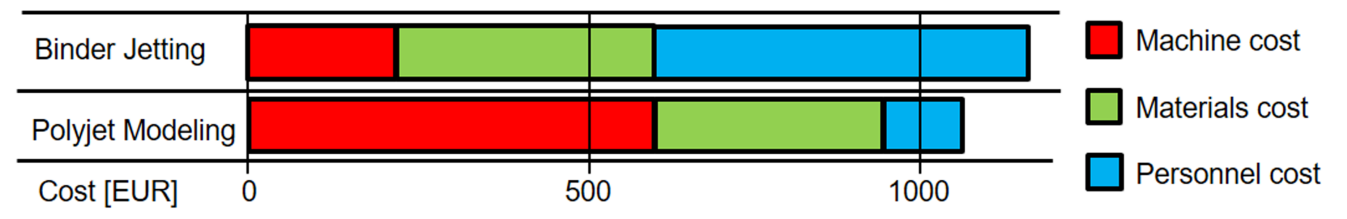

in this comparison. The printing time has an important influence on the printing costs. This differs significantly in both methods. While in the BJ a 3D printing time of about only 6.6 hours for the house model, the $3 \mathrm{D}$ printing of the house using PJM method required more than 36 hours in total. In materials cost, volume is the most important influencing factor. The volume of the house amounts to approximately 384 cubic centimeters. For personnel costs, above all the time required for reworking, ie the removal of superfluous powder (BJ) or the support structures (PJM), is important. This time expenditure lies with the house model for BJ with approximately 7 hours, with the PJM only 2.7 hours. Figure 5 illustrates the cost of the house model. The cost are calculated as the average of several offers. It shows that the costs for PJM models, despite the long production time, are slightly lower than the BJ method.

\section{DISCUSSION}

This contribution shows that architectural models can be produced using modern digital manufacturing techniques. The necessary 3D models can be derived with only little effort directly from the CAAD software by hiding unprintable areas. In addition, a seperation into floors and terrain model, which are printed separately is possible. The examination of the preprocessing has shown that various software packages are available for repairing the 3D data and for setting the printing parameters. Here a suitable choice of the interfaces and data formats for the data transfer has to be considered. 3D printing provides very pleasing results in both methods. In the cost analysis, the costs for 3D printing, materials and reworking were analyzed. This case study showed a slight cost advantage for the PJM process.

\section{ACKNOWLEDGMENTS}

We would like to thank the architects IFP2 Hangs $\mathrm{GmbH}$ in Lahr for their generous support in this contribution. In particular, the provision of practicerelevant CAAD data and the extensive feedback on the results have made the present work possible.

\section{REFERENCES}

Gibson, I, Kvam, T and Wai Ming, L 2002, 'Rapid prototyping for architectural models', Rapid Prototyping Journal, 8 (2), p. 91-95

Hull, C and Willet, W 2017 'Building with Data: Architectural Models as Inspiration for Data Phyicalization', Proceedings of CAHI 2017, pp. 1217-1228

Junk, S and Gawron, P 2018, 'Implementation of Innovative Methods for the Digital Manufacturing of Architectural Models', Int. Journal of Contemporary Architecture "The New ARCH", Vol. 5, No. 2, pp. 1-8

Mancanares, CG, Zancul, E, Cavalcante da Silva, J and Cauchick, MPA 2015, 'Additive manufacturing process selection based on parts' selection criteria', Int $J$ Adv Manuf Technol, 80 (5-8), p. 1007-1014

Meijs, P 2014, Rapid Prototyping and 3D Printing in Architecture, Blurb books

Ryder, G, lon, B, Green, G, Harrison, D and Wood, B 2002, 'Rapid design and manufacture tools in architecture', Automation in Construction, 11 (3), p. 279-290

Stavric, M, Siddanin, P and Tepavcevic, B 2013, Architectural Scale Models in the Digital Age: Design, Representation and Manufacturing, Springer

Wohlers, T 2017, Wohlers report 2017. 3D printing and additive manufacturing state of the industry annual worldwide progress report. Fort Collins, Col.: Wohlers Associates., Wohlers Associates

Wong, KV and Hernandez, A 2012, 'A Review of Additive Manufacturing', ISRN Mechanical Engineering, (4), pp. $1-10$

Zegard, T and Paulino, GH 2016, 'Bridging topology optimization and additive manufacturing', Struct Multidisc Optim, 53 (1), p. 175-192
Figure 5

Cost for

architectural model of detached house (scale 1:100) using Binder Jetting and Polyjet Modelling 